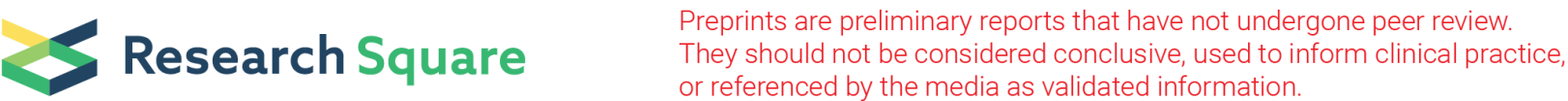

\section{Use of a New Off-the-shelf 3D-printed Trabecular Titanium Acetabular Cup in Chinese Patients Undergoing Hip Revision Surgery: Short- to Mid- term Clinical and Radiological Outcomes}

\section{Guangqian Shang}

The Affiliated Hospital of Qingdao University

\section{Shuai Xiang}

The Affiliated Hospital of Qingdao University

\section{Cuicui Guo}

The Affiliated Hospital of Qingdao University

Jianjun Guo

The Affiliated Hospital of Qingdao University

\section{Peng Wang}

The Affiliated Hospital of Qingdao University

\section{Yingzhen Wang}

The Affiliated Hospital of Qingdao University

Hao Xu ( $\nabla 18661806627 @ 163 . c o m$ )

The Affiliated Hospital of Qingdao University

\section{Research Article}

Keywords: Reoperation, Hip, Trabecular titanium acetabular cup, 3D printing

Posted Date: November 15th, 2021

DOl: https://doi.org/10.21203/rs.3.rs-900433/v2

License: (c) (i) This work is licensed under a Creative Commons Attribution 4.0 International License. Read Full License 


\section{Abstract}

Background: Revision total hip arthroplasty (THA) has been a challenge for surgeons. The purpose of this study was to explore the short-to mid-term clinical and radiological outcomes of Chinese patients who underwent revision THA using a new off-the-shelf three-dimensional (3D)-printed trabecular titanium (TT) acetabular cup by comparison with a conventional porous coated titanium acetabular cup, to provide a reference for the recommendation of this prostheses.

Methods: A retrospective analysis of 57 patients ( 57 hips) who received revision THA was performed from January 2016 to June 2019. A total of 23 patients received 3D-printed cups (observation group) and 34 patients received non-3D-printed cups (control group). Clinical scores including Visual Analogue Scale (VAS), Harris Hip Score (HHS) and Short Form 36 (SF-36), upward movement of the hip center of rotation \HCOR $\$ and limb-length discrepancy (LLD), stabilization and bone ingrowth of cups were compared between two groups. The multivariate linear regression was used to determine the factors potentially influencing the HHS score. Postoperative complications in the two groups were also recorded.

Results: All 57 patients were routinely followed up. The average follow-up durations in the control and observation groups were $43.57 \pm 13.68$ (24-65) months and $41.82 \pm 11.44$ (24-64) months, respectively $(p=0.618)$. The postoperative clinical scores significantly improved in both groups compared to the preoperative scores $(p<0.05)$. The VAS score did not significantly differ between the groups at 3 or 12 months postoperatively, or at the last follow-up $(p>0.05)$. The HHS and SF-36 scores did not significantly differ between the groups at 3 months postoperatively $(p>0.05)$ but differed at 12 months postoperatively and the last follow-up $(p<0.05)$. Compared with the control group, the postoperative recovery of HCOR and LLD was better in the observation group $(p<0.05)$. All cups remained stable, with no loosening throughout the follow-up period. But the observation group had a significantly better rate of bone ingrowth compared to the control group $(p=0.037)$. Multivariate linear regression analysis showed that different cup types, upward movement of the HCOR, and LLD influenced the HHS score at the last follow-up $(p<0.05)$. None of the patients exhibited severe postoperative complications.

Conclusion: The new off-the-shelf 3D-printed TT acetabular cup demonstrated encouraging short-to midterm clinical outcomes in Chinese patients. It can effectively relieve pain, improve hip function, provide satisfactory biological fixation and high survival rate. But further follow up is necessary to assess its long-term outcomes.

\section{Background}

Total hip arthroplasty (THA) is an effective surgery for end-stage hip diseases, such as avascular necrosis of femoral heads and osteoarthritis, because it relieves pain and improves joint function and quality of life [1]. Although the longevity of prostheses has significantly improved because of advancements in surgical techniques and prosthesis design, they all need to be replaced over time [2]. Because of the aging population and primary THA being performed in younger patients, the number of 
revision surgeries is gradually increasing. It is estimated that the number of revision THAs performed in the USA will double by 2026 [3].

The most common indication for revision THA is aseptic loosening (AL) of acetabular cups [4]. One of the major objectives of revision surgery is early and long-term stable fixation of implants. Acetabular bone defects are also a challenge for surgeons, even the most experienced ones. These defects can affect primary mechanical stability and cause secondary bone ingrowth, which may necessitate re-revision surgery. The Paprosky classification, based on the integrity of Kohler's line, teardrop or ischium osteolysis, and acetabular component migration, is the most commonly used classification system [5]. A study of 1,094 cases of revision THA reported severe acetabular bone defects in $17 \%$ of cases, but the surgical failure rate was as high as $30 \%[6]$.

In recent years, three-dimensional (3D) printing technology has been widely used in medical fields, especially orthopedics [7]. Traditionally, metal implants were created by "formative shaping" or "subtractive manufacturing"; however, 3D printing technology has made additive manufacturing a reality. Electron beam melting (EBM), an important 3D printing technology, allows titanium to be melted at nearly $2,000^{\circ} \mathrm{C}$ and produces acetabular cups with a specific design and controlled porous surface. These cups reduce the elastic modulus and have biological characteristics similar to those of subchondral bone [8,9].

In this study of Chinese patients who underwent revision surgery, we compared a new off-the-shelf 3Dprinted trabecular titanium (TT) cup to a conventional titanium cup with a porous coated back.

\section{Methods}

\section{Inclusion and exclusion criteria}

After the study had been approved by our Institutional Review Board (QYFYWZLL-26263), we retrospectively collected data from 57 patients ( 57 hips) who underwent revision THA between January 2016 and June 2019 at the Affiliated Hospital of Qingdao University (Qingdao, China). We included patients who were diagnosed with AL or periprosthetic joint infection (PJI) after unilateral primary THA. We excluded patients with insufficient neuromuscular function, as this may cause postoperative hip instability or gait abnormality, periprosthetic fracture after primary THA due to trauma, uncontrolled PJI, or infections other than PJI.

\section{Acetabular implants}

Based on the acetabular revision material used, patients were divided into two groups. Patients who received a 3D-printed TT acetabular cup (Aikang Corp., Beijing, China) were included in the observation group (Figure 1). Because of the continuity between the metal solid and surface porous layers, it provided greater resistance against detachment and corrosion. The implant had a 1.5-mm-thick trabecular-like porous titanium construct on the back surface, with an average porosity of $80 \%$ and pore size of $600-$ $1,000 \mu \mathrm{m}$. Patients who received a conventional porous titanium-coated acetabular cup (Reflection 
Acetabular System, Smith and Nephew, Memphis, TN, USA), were included in the control group. This implant had a porous coating of sintered titanium beads.

\section{Surgical procedures}

Patients received general anesthesia in combination with fascial iliac block. All revision surgeries were performed by three senior surgeons, using a modified Hardinge approach. After exposure, the original prostheses or spacers, periacetabular scar tissues, cement, and particles were thoroughly removed. The degree of acetabular bone defect was assessed by direct observation. The acetabular rotation center was identified, and the acetabulum was sequentially reamed using dedicated hemispherical reamers until blood oozed evenly from the bone surface. The bone defect was reassessed in terms of the need for metal augment or structural bone graft to fill the cavitary defects, and trial implants were inserted to assess coverage, impingement, and stability. In cases of severe acetabular bone defects, metal augment or structural bone graft from autologous iliac bone was used to fill the defect between the cup's dome and host bone, followed by screws to ensure stability of augment or bone graft. According to the preoperative plan, the definitive cup was inserted at the appropriate abduction $\left(40 \pm 10^{\circ}\right)$ and anteversion $\left(15 \pm 5^{\circ}\right)$ angles. The cup was stabilized by $1-3$ screws inserted through the holes. After replacing the cups, stems were also revised if loosening was observed on preoperative imaging studies or intraoperatively.

\section{Postoperative recovery}

Multimodal analgesia was administered to the patients according to clinical guidelines. The analgesia included intravenous flurbiprofen axetil and oral oxycodone. Cephalosporins were administered for $48 \mathrm{~h}$ postoperatively to prevent infections; patients who were allergic to cephalosporin were administered clindamycin. Rivaroxaban ( $25 \mathrm{mg} /$ day) was given orally within 12-24 $\mathrm{h}$ after the operation, and continued for a month to reduce the risk of deep venous thrombosis. Functional exercises were gradually introduced over time. During the first 6 weeks postoperatively, patients dependent on crutches were not permitted to bear weight on the affected limbs; however, partial weight bearing was allowed from 6 weeks to 3 months, and full weight bearing after 3 months.

\section{Clinical and radiographic assessment}

Visual analog scale (VAS), Harris Hip Score (HHS), and Short-Form 36 (SF-36) values were recorded preoperatively, 3 and 12 months postoperatively, and at the last follow-up for patients in both groups. These assessments were performed by the same clinical staff, who did not participate in the surgery.

Anteroposterior hip joint radiographs were obtained to evaluate upward movement of the hip center of rotation (HCOR) and limb-length discrepancy (LLD) preoperatively, 1 day postoperatively, and at the last follow-up. Bilateral teardrop connection was used as the reference line for these assessments; the upward movement distance of HCOR was defined as the vertical distance between HCOR on the affected side 
and the reference line, and LLD was defined as the difference in vertical distance between the vertices of bilateral lesser trochanters and the reference line.

Using the zonal analysis of DeLee and Charnley [10], cup position was evaluated according to the width of the radiolucent line (RLL) and changes in abduction angle and displacement distance. Cup stabilization was defined as the width of a RLL less than $1 \mathrm{~mm}$ in two zones, no RLL in at least two zones, and no displacement. Cup loosening was defined as a change in abduction angle of more than $10^{\circ}$ or cup migration of more than $6 \mathrm{~mm}$ in any direction. Bone ingrowth of cups was evaluated according to the criteria defined by the Anderson Orthopedic Research Institute [11]. Osseointegration was diagnosed if at least three of the following signs were observed: absence of RLLs; presence of superolateral buttresses; presence of medial stress-shielding; presence of radial trabeculae; and presence of inferomedial buttresses.

Postoperative complications in the two groups were also recorded.

\section{Statistical analyses}

Statistical analyses were performed using SPSS software (version 24.0; IBM Corp., Armonk, NY, USA). The Shapiro-Wilk test was used to determine the normality of the data. Continuous variables are expressed as means and standard deviations (SDs). Student's t-test was used to compare continuous variables between the two groups. Clinical scores, as well as upward movement of the HCOR and LLD, were compared between groups using analysis of variance. Multiple comparisons were made using the least significant difference (LSD) test. Categorical variables are presented as frequencies and constituent ratios, and were compared using the $\chi^{2}$ test. Multivariate linear regression was used to determine the factors that potentially influenced the HHS score. $\mathrm{P}<0.05$ indicated statistical significance.

\section{Results}

\section{Demographic characteristics}

Twenty-three patients received 3D-printed cups (observation group) and 34 received non-3D-printed cups (control group). No patients were lost to follow up, and 57 patients were routinely followed up either in the outpatient department or over the telephone. The average follow-up durations in the control and observation groups were $43.57 \pm 13.68$ (24-65) months and $41.82 \pm 11.44$ (24-64) months, respectively. The acetabular defects were graded according to the Paprosky classification: 4 were Paprosky I, 9 Paprosky Ila, 5 Paprosky Ilb, 1 Paprosky Ilc, and 4 Paprosky IIla in the observation group, whereas 6 were Paprosky I, 13 Paprosky Ila, 8 Paprosky Ilb, 2 Paprosky Ilc, and 5 Paprosky IIla in the control group. To repair the bone defect, four patients in the observation group required augment graft, one required structural bone graft from the ilium, and five required combined augment and structural bone grafts. In the control group, six patients required augment graft, three required structural bone graft, and six required combined augment and structural bone grafts. 
As shown in Table 1, no significant differences were observed in age, body mass index (BMI), gender, laterality, mean follow-up duration, reason for revision surgery, or Paprosky classification between the two groups ( $p>0.05)$. Figures 2 and $\mathbf{3}$ present representative images of 3D-printed TT acetabular cups.

\section{Clinical scores}

Figure 4 depicts the clinical scores for both groups. The postoperative VAS, HHS, and SF-36 scores significantly improved in both groups compared to the preoperative scores $(p<0.05)$. The VAS score did not significantly differ between the groups at $3(p=0.946)$ or $12(p=0.681)$ months postoperatively, or at the last follow-up ( $p=0.885)$. The HHS score did not significantly differ between the groups at 3 months $(p=0.378)$ postoperatively, but was significantly different at 12 months $(p<0.001)$ postoperatively and the last follow-up ( $<$ 0.001). The SF-36 score did not significantly differ between the groups at 3 months $(p=0.289)$ postoperatively, but was significantly different at 12 months $(p<0.001)$ postoperatively and the last follow-up $(p<0.001)$.

\section{HCOR and LLD}

Figure 5 depicts the upward movement of the HCOR and LLD in both groups. In both groups, these values were significantly lower at 1 day postoperatively and the last follow-up compared to the preoperative values $(p<0.05)$. However, postoperative recovery was better in the observation group $(p<0.05)$.

\section{Stabilization and bone ingrowth of cups}

All cups remained stable, with no loosening throughout the follow-up period. The cup abduction angle did not change between 1 day postoperatively and the last follow-up in the observation $(p=0.888)$ or control $(p=0.963)$ group (Table 2). In the observation group, radiographs revealed that the three cups had 2-mm RLLs in zones I and II at 1 day postoperatively. These RLLS disappeared in two cups at 3 months postoperatively, and in one cup at 12 months postoperatively. In the control group, five cups had 1-mm RLLs in zones I and II, and three cups had 2-mm RLLs in zones II and III at 1 day postoperatively. Only three cups had 1-mm RLLs that did not disappear, but fortunately these also showed no progression. All other RLLs disappeared at 12 months postoperatively. No new RLLS appeared in the patients during the follow-up period.

Based on the bone ingrowth criteria, 2 cups in the observation group had two signs, 17 had three signs, and 4 had four signs preoperatively. However, postoperatively, 10 cups had two signs, 22 had three signs, and 2 had four signs at the last follow-up. The observation group had a significantly better rate of bone ingrowth compared to the control group ( $91.30 \%$ and $70.59 \%$, respectively; $p=0.037$ ) (Table 3).

\section{Factors potentially influencing the HHS score at the last follow-up}

Multivariate linear regression analysis showed that different cup types, upward movement of the HCOR influenced the HHS score at the last follow-up ( $p<0.001, p=0.005$, respectively), whereas age, gender, BMI, laterality, and the preoperative HHS score did not ( $p>0.05)$ (Table 4). 


\section{Complications}

None of the patients exhibited PJI, loosening, dislocation, deep venous thrombosis, or nerve palsy. One patient in the observation group and three in the control group had poor wound healing with oozing, which was resolved by timely dressing change and prolonged antibiotic use. In addition, one patient in the observation group and two in the control group had mild persistent pain, leading to anxiety, for 24 months postoperatively. This pain was not effectively treated with non-steroidal anti-inflammatory drugs.

\section{Discussion}

How to improve the efficacy of revision THA has always been a hot-spot issue. Re-revision after failed revision surgery is not only an extremely complex operation with long treatment duration, many postoperative complications, and a high mortality rate, but also places great physiological, psychological, and economic burdens on the patient. A review of the literature confirmed that this is the first study to assess the new 3D-printed TT acetabular cup for use in revision THA. The results showed that this acetabular component is a reliable option for revision surgery, even in cases with severe acetabular bone loss.

Baauw et al. [12] reported that a cage is still the most frequently used technique in revision surgery. A cage that spans the ilium and ischia reconstructs the bone and provides initial stability, but does not support bone ingrowth or long-term biological fixation; in the long term, cages eventually break down or loosen due to fatigue $[13,14]$. There has been a steady increase in the use of porous titanium customized acetabular implants in revision THA. These implants provide an individualized fit for each patient and achieve satisfactory results, including improvement in hip joint function and good stability even with severe acetabular bone loss or pelvic discontinuity [15-17]. However, customized implants are not indispensable if bone loss is not severe.

An important feature of EBM is the realization of off-the-shelf 3D-printed TT acetabular cups. These cups have the advantages of decreased economic burden and reduced preoperative preparation duration compared to customized implants. The 3D-printed TT acetabular cups can be used alone for mild bone loss, or in combination with other surgical approaches for severe bone loss. Satisfactory clinical and radiographic outcomes of off-the-shelf 3D-printed TT cups in primary THA have also encouraged their use in revision surgeries. The DELTA-TT cup (Lima Corp., Udine, Italy) is manufactured by EBM and has a highly porous, hemispherical, multi-hole design, similar to Aikang TT cups. Geng et al. [18] reported excellent results in a cohort of 92 patients who underwent primary THA using DELTA-TT cups after a mean follow-up duration of 48.2 months. The survival rate of the cups was $100 \%$, with no signs of cup loosening. Perticarini et al. [19] studied 133 cases of primary or revision THA using DELTA-TT cups and reported similar results, i.e., a $99.5 \%$ survival rate and excellent functional scores after a minimum of 5 years of follow-up. They suggested that this implant is optimal for primary surgery in cases of severe acetabular disease. 
In the present study, although both cup types effectively alleviated pain, the 3D-printed cup was associated with higher HHS and SF-36 scores. A similar outcome was reported by Wan et al. [20] in a small series of 22 patients with 3D-printed cups and 20 patients with non-3D-printed cups. They reported that patients in the 3D-printed group achieved better functional scores, including HHS and SF-36, at 3, 6, and 12 months postoperatively. This difference was closely related to the HCOR and LLD [21]. One of main aims of surgery is to restore the normal HCOR and LLD. Excessive HCOR affects the stress distribution in soft tissues around the hip, causing decreased strength of the gluteus medius and adductor muscles, resulting in hip joint dysfunction, dislocation, and accelerated wear of the polyethylene liner, which has a significant impact on the early and long-term survival of the prosthesis [21]. Better postoperative recovery in our 3D-printed group was confirmed on the basis of upward movement of the HCOR in radiographs. The multivariate linear regression analysis also confirmed that upward movement of the HCOR influenced the HHS score at the last follow-up.

Initial stability and secondary bone growth of cups were also evaluated in this study. Although loosening did not occur with either 3D-printed or conventional cups, a significant difference was observed in the rate of bone ingrowth between the two groups ( $91.30 \%$ and $70.59 \%$, respectively) due to the highly porous surface and higher friction coefficient of the 3D-printed cups. TT, which consists of interconnected cells that form multi-planar hexagons, is a highly porous honeycomb structure that can mimic the trabecular morphology of human bone [22]. Many in intro studies reported that TT produced by EBM has good osteoinductive properties that stimulate vascularization, and osteoblast proliferation and differentiation $[23,24]$. A sheep model study conducted by Declan et al. [25] demonstrated that, compared to traditional porous coatings, TT provided an optimized microenvironment for bone growth.

Melancon et al. [26] reported that the porosity and pore size of TT were key factors affecting bone growth, by affecting the transmission of fluids and providing a favorable microenvironment for the attachment and proliferation of osteoblasts. Additionally, the rough morphology of the pores increased the surface area for cell attachment and improved the surface energy of the material. Human trabecular bone has a porosity of $50-90 \%$ and pore size of $1,000 \mu \mathrm{m}$, and is an interconnected and open-porous structure. A porosity of $60-80 \%$ and pore size of $300-1,200 \mu \mathrm{m}$ are suitable for cell growth and proliferation [27]. Porosity and pore size can be freely regulated and controlled using EBM during the additive manufacturing process. Three previous studies reported excellent stability and bone growth of the DELTATT cup, with an average porosity of $65 \%$ and mean pore size of $640 \mu \mathrm{m}$. De Meo et al. [28] observed no RLLs or signs of migration in 52 patients who underwent revision using Delta TT cups after a mean follow-up of 48.3 months, except in 6 patients who underwent re-revision. Relatively poorer outcomes were achieved by Gallart et al. [21] in 67 revision patients, and by Steno et al. [29] in 81 revision patients. In this study, an Aikang cup with an average porosity of $80 \%$ and pore size of $600-1,000 \mu \mathrm{m}$ showed better bone growth, with no patients requiring re-revision for $\mathrm{AL}$.

In addition to the aforementioned advantages, Dall'Ava et al. [30] revealed that the existence of titanium beads on three types of off-the-shelf 3D-printed TT cups was a by-product of the manufacturing process, where the beads may be released in the body. Bistolfi et al. [31] reported no difference in body titanium 
levels, as measured in blood and urine samples, between 3D-printed and conventional titanium cups. Research on this topic is limited. It is unclear whether titanium adversely affects clinical outcomes; more research is needed.

This study had several limitations. First, it was a retrospective, single center study. We plan to conduct a multicenter, prospective randomized controlled trial in the future to generate higher-level evidence. Another limitation was the inadequate follow-up duration. Long-term follow-up is indispensable for the assessment of clinical and radiological outcomes, complications, and cup survival rates.

\section{Conclusion}

The new off-the-shelf 3D-printed TT acetabular cup demonstrated encouraging short-to mid-term clinical outcomes in Chinese patients. It can effectively relieve pain, improve hip function, provide satisfactory biological fixation and high survival rate. But further follow up is necessary to assess its long-term outcomes.

\section{Abbreviations}

THA, total hip arthroplasty; AL, aseptic loosening; 3D, three-dimensional; EBM, Electron beam melting; TT, trabecular titanium; PJI, periprosthetic joint infection; HCOR, hip center of rotation; LLD, limb-length discrepancy; RLL, radiolucent line

\section{Declarations}

\section{Ethics approval and consent to participate}

This study was approved by Medical Ethics Committee of Affiliated Hospital of Qingdao University. Informed consent was obtained from all individual participants included in the study. All procedures performed in studies involving human participants were in accordance with the ethical standards of the institutional research committee and with the 1964 Helsinki declaration and its later amendments or comparable ethical standards

\section{Consent for publication}

Written informed consent was obtained from the patient for publication of this case report and any accompanying images. A copy of the written consent is available for review by the Editor of this journal.

\section{Availability of data and materials}

The final dataset will be available from the corresponding author.

\section{Competing interests}


The authors declare that they have no competing interests.

\section{Funding}

The study is supported by grants from National Natural Science Foundation of China (82002349). These funding bodies had role in the collection and interpretation of data and in writing the manuscript.

\section{Authors' contributions}

GS: wrote the paper. SX, CG, and JG: collected the data and participated in writing and revising the paper. PW and YW: performed all the analysis. HX: designed the whole study.

\section{Acknowledgements}

N/A.

\section{Authors' information}

1 Department of Joint Surgery, The Affiliated Hospital of Qingdao University, No. 59, Haier Road, Qingdao 266000, China.

\section{References}

1. Kehlet H: Fast-track hip and knee arthroplasty. Lancet 2013, 381(9878):1600-1602.

2. Van Kleunen JP, Lee GC, Lementowski PW, Nelson CL, Garino JP: Acetabular revisions using trabecular metal cups and augments. J Arthroplasty 2009, 24(6 Suppl):64-68.

3. Weber M, Witzmann L, Wieding J, Grifka J, Renkawitz T, Craiovan B: Customized implants for acetabular Paprosky III defects may be positioned with high accuracy in revision hip arthroplasty. Int Orthop 2019, 43(10):2235-2243.

4. Haenle M, Podbielski A, Mittelmeier W, Bader R, Gradinger R, Gollwitzer H: Infections after primary and revision total hip replacement caused by enterobacteria producing extended spectrum betalactamases (ESBL): a case series. Hip Int 2010, 20(2):248-254.

5. Paprosky WG, Perona PG, Lawrence JM: Acetabular defect classification and surgical reconstruction in revision arthroplasty. A 6-year follow-up evaluation. J Arthroplasty 1994, 9(1):33-44.

6. Johanson NA, Driftmier KR, Cerynik DL, Stehman CC: Grading acetabular defects: the need for a universal and valid system. J Arthroplasty 2010, 25(3):425-431.

7. Wong KC: 3D-printed patient-specific applications in orthopedics. Orthop Res Rev 2016, 8:57-66.

8. Marin E, Fusi S, Pressacco M, Paussa L, Fedrizzi L: Characterization of cellular solids in Ti6Al4V for orthopaedic implant applications: Trabecular titanium. J Mech Behav Biomed Mater 2010, 3(5):373381. 
9. Dallago M, Fontanari V, Torresani E, Leoni M, Pederzolli C, Potrich C, Benedetti M: Fatigue and biological properties of Ti-6Al-4V ELI cellular structures with variously arranged cubic cells made by selective laser melting. J Mech Behav Biomed Mater 2018, 78:381-394.

10. DeLee JG, Charnley J: Radiological demarcation of cemented sockets in total hip replacement. Clin Orthop Relat Res 1976(121):20-32.

11. Moore MS, McAuley JP, Young AM, Engh CA, Sr.: Radiographic signs of osseointegration in porouscoated acetabular components. Clin Orthop Relat Res 2006, 444:176-183.

12. Baauw M, van Hooff ML, Spruit M: Current Construct Options for Revision of Large Acetabular Defects: A Systematic Review. JBJS Rev 2016, 4(11).

13. Regis D, Magnan B, Sandri A, Bartolozzi P: Long-term results of anti-protrusion cage and massive allografts for the management of periprosthetic acetabular bone loss. J Arthroplasty 2008, 23(6):826-832.

14. Berry DJ: Antiprotrusio cages for acetabular revision. Clin Orthop Relat Res 2004(420):106-112.

15. Burastero G, Cavagnaro L, Chiarlone F, Zanirato A, Mosconi L, Felli L, de Lorenzo FDR: Clinical study of outcomes after revision surgery using porous titanium custom-made implants for severe acetabular septic bone defects. Int Orthop 2020, 44(10):1957-1964.

16. Chiarlone F, Zanirato A, Cavagnaro L, Alessio-Mazzola M, Felli L, Burastero G: Acetabular custommade implants for severe acetabular bone defect in revision total hip arthroplasty: a systematic review of the literature. Arch Orthop Trauma Surg 2020, 140(3):415-424.

17. Fröschen FS, Randau TM, Hischebeth GTR, Gravius N, Gravius S, Walter SG: Mid-term results after revision total hip arthroplasty with custom-made acetabular implants in patients with Paprosky III acetabular bone loss. Arch Orthop Trauma Surg 2020, 140(2):263-273.

18. Geng X, Li Y, Li F, Wang X, Zhang K, Liu Z, Tian H: A new 3D printing porous trabecular titanium metal acetabular cup for primary total hip arthroplasty: a minimum 2-year follow-up of 92 consecutive patients. J Orthop Surg Res 2020, 15(1):383.

19. Perticarini L, Zanon G, Rossi SM, Benazzo FM: Clinical and radiographic outcomes of a trabecular titanium ${ }^{\mathrm{m}}$ acetabular component in hip arthroplasty: results at minimum 5 years follow-up. $B M C$ Musculoskelet Disord 2015, 16:375.

20. Wan L, Wu G, Cao P, Li K, Li J, Zhang S: Curative effect and prognosis of 3D printing titanium alloy trabecular cup and pad in revision of acetabular defect of hip joint. Exp Ther Med 2019, 18(1):659663.

21. Gallart X, Fernández-Valencia JA, Riba J, Bori G, García S, Tornero E, Combalía A: Trabecular TitaniumTM cups and augments in revision total hip arthroplasty: clinical results, radiology and survival outcomes. Hip Int 2016, 26(5):486-491.

22. Imai H, Miyawaki J, Kamada T, Maruishi A, Takeba J, Miura H: Radiolucency around highly porous sockets and hydroxyapatite-coated porous sockets in total hip arthroplasty for hip dysplasia. Eur $J$ Orthop Surg Traumatol 2019, 29(3):611-618. 
23. Wang C, Xu D, Li S, Yi C, Zhang X, He Y, Yu D: Effect of Pore Size on the Physicochemical Properties and Osteogenesis of Ti6Al4V Porous Scaffolds with Bionic Structure. ACS Omega 2020, 5(44):28684-28692.

24. Gastaldi G, Asti A, Scaffino MF, Visai L, Saino E, Cometa AM, Benazzo F: Human adipose-derived stem cells (hASCs) proliferate and differentiate in osteoblast-like cells on trabecular titanium scaffolds. J Biomed Mater Res A 2010, 94(3):790-799.

25. IN VIVO EVALUATION OF THE OSTEOINTEGRATION OF NEW HIGHLY POROUS TRABECULAR TITANIUM\&\#8482. Orthopaedic Proceedings 2012, 94-B:201-201.

26. Melancon D, Bagheri ZS, Johnston RB, Liu L, Tanzer M, Pasini D: Mechanical characterization of structurally porous biomaterials built via additive manufacturing: experiments, predictive models, and design maps for load-bearing bone replacement implants. Acta Biomater 2017, 63:350-368.

27. Teixeira LN, Crippa GE, Lefebvre LP, De Oliveira PT, Rosa AL, Beloti MM: The influence of pore size on osteoblast phenotype expression in cultures grown on porous titanium. Int J Oral Maxillofac Surg 2012, 41(9):1097-1101.

28. De Meo F, Cacciola G, Bellotti V, Bruschetta A, Cavaliere P: Trabecular Titanium acetabular cups in hip revision surgery: mid-term clinical and radiological outcomes. Hip Int 2018, 28(2_suppl):61-65.

29. Steno B, Kokavec M, Necas L: Acetabular revision arthroplasty using trabecular titanium implants. Int Orthop 2015, 39(3):389-395.

30. Dall'Ava L, Hothi H, Henckel J, Di Laura A, Shearing P, Hart A: Comparative analysis of current 3D printed acetabular titanium implants. 3D Print Med 2019, 5(1):15.

31. Bistolfi A, Cimino A, Lee GC, Ferracini R, Maina G, Berchialla P, Massazza G, Massè A: Does metal porosity affect metal ion release in blood and urine following total hip arthroplasty? A short term study. Hip Int 2018, 28(5):522-530.

\section{Tables}

Table 1 Demographic data of patients ( $M \pm S D s$ ) 
Observation group Control group $(n=34) \quad P$ value $(n=23)$

\begin{tabular}{lcll} 
Age (year) & $70.35 \pm 8.10$ & $71.62 \pm 10.23$ & 0.604 \\
\hline BMI (Kg/ m${ }^{2}$ ) & $25.61 \pm 2.80$ & $26.26 \pm 2.49$ & 0.360 \\
\hline Gender (number, \%) & & & 0.791 \\
\hline Male & $10,43.48$ & $14,41.18$ & \\
\hline Female & $13,56.52$ & $20,58.82$ & 0.550 \\
\hline Laterality (number, \%) & & & \\
\hline Left hip & $11,47.83$ & $15,44.12$ & 0.605 \\
\hline Right hip & $12,52.17$ & $19,55.88$ & 0.684 \\
\hline Mean follow-up time (month) & $43.57 \pm 13.68$ & $41.82 \pm 11.44$ & \\
\hline Reason for revision (number, \%) & & & \\
\hline Aseptic loosening & $17,73.91$ & $24,70.59$ & \\
\hline Infection & $6,26.09$ & $10,29.41$ & \\
\hline Paprosky classification (number, \%) & & \\
\hline Type I & $4,17.39$ & $6,17.65$ & \\
\hline Type II (A+B+C) & $15,65.22$ & $23,67.65$ & $5,14.70$ \\
\hline Type III (A+B) & $4,17.39$ & & \\
\hline
\end{tabular}

$\mathrm{M} \pm$ SDs: mean \pm standard deviations, BMI: body mass index

Table 2 The cup abduction angle between the observation and control groups $(M \pm S D s)$

\begin{tabular}{|llll|}
\hline & 1 day postoperatively & the last follow-up & P value \\
\hline Observation group & $39.35 \pm 5.41$ & $39.12 \pm 5.40$ & 0.888 \\
\hline Control group & $40.07 \pm 5.51$ & $40.14 \pm 5.40$ & 0.963 \\
\hline P value & 0.625 & 0.490 & - \\
\hline
\end{tabular}

$\mathrm{M} \pm$ SDs: mean \pm standard deviation

Table 3 Bone ingrowth between the observation and control groups at the last follow-up 


\begin{tabular}{|c|c|c|c|c|}
\hline & \multicolumn{2}{|c|}{ bone ingrowth criteria } & \multirow[t]{2}{*}{ Total } & \multirow[t]{2}{*}{ Rate of bone ingrowth } \\
\hline & $\otimes 3$ signs & $\geq 3$ signs & & \\
\hline Observation group & 2 & 21 & 23 & $91.30 \%$ \\
\hline Control group & 10 & 24 & 34 & $70.59 \%$ \\
\hline Total & 12 & 45 & 57 & $78.95 \%$ \\
\hline
\end{tabular}

At least 3 signs can be considered as good bone ingrowth. The $\chi^{2}$ test was used for statistical analysis: $\chi^{2}=3.121, P=.037$.

Table 4 Multivariate linear regression of factors on the HHS score at the last follow-up

\begin{tabular}{|llll|}
\hline & Beta & P value & VIF \\
\hline Age & 0.022 & 0.806 & 1.122 \\
\hline Gender & 0.454 & 0.065 & 8.028 \\
\hline BMI & 0.026 & 0.778 & 1.217 \\
\hline Laterality & -0.429 & 0.083 & 8.199 \\
\hline the HHS score before surgery & 0.071 & 0.451 & 1.228 \\
\hline 3D printed cups vs non-3D printed cups & -0.603 & $<0.001$ & 2.427 \\
\hline The upward movement of HCOR at the last follow-up & -0.324 & 0.005 & 1.711 \\
\hline
\end{tabular}

HHS: Harris Hip Score, VIF: Variance Inflation Factor, BMI: body mass index, HCOR: hip center of rotation

\section{Figures}


\section{Figure 1}

The pictures showed the appearance of three-dimensional printed trabecular titanium acetabular cup (A) and the SEM image of its interconnected trabecular titanium cellular solid structure (B). SEM: scanning electron microscope 

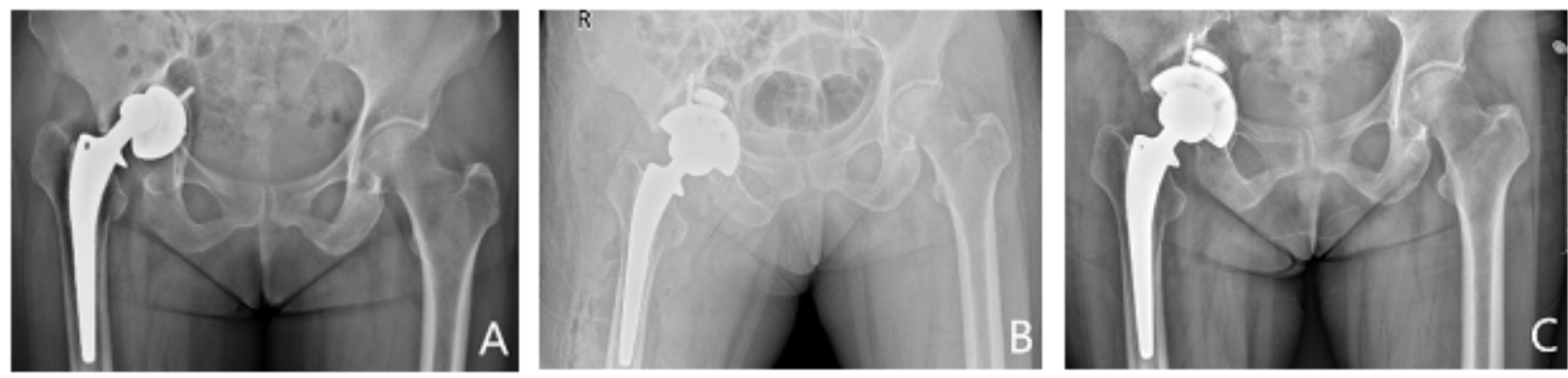

\section{Figure 2}

Fourteen years after primary total hip arthroplasty, a 69-year-old woman underwent revision surgery using a three-dimensional printed trabecular titanium acetabular cup combined with metal augment and structural bone due to aseptic loosening. (A) Pre-operative radiograph showing a large acetabular defect. (B) Radiograph obtained at 1 day postoperatively showed that the appropriate hip center of rotation had been restored. (C) Radiograph obtained at 30 months postoperatively showed that the cup and augment were stable, and the bone graft was incorporated.
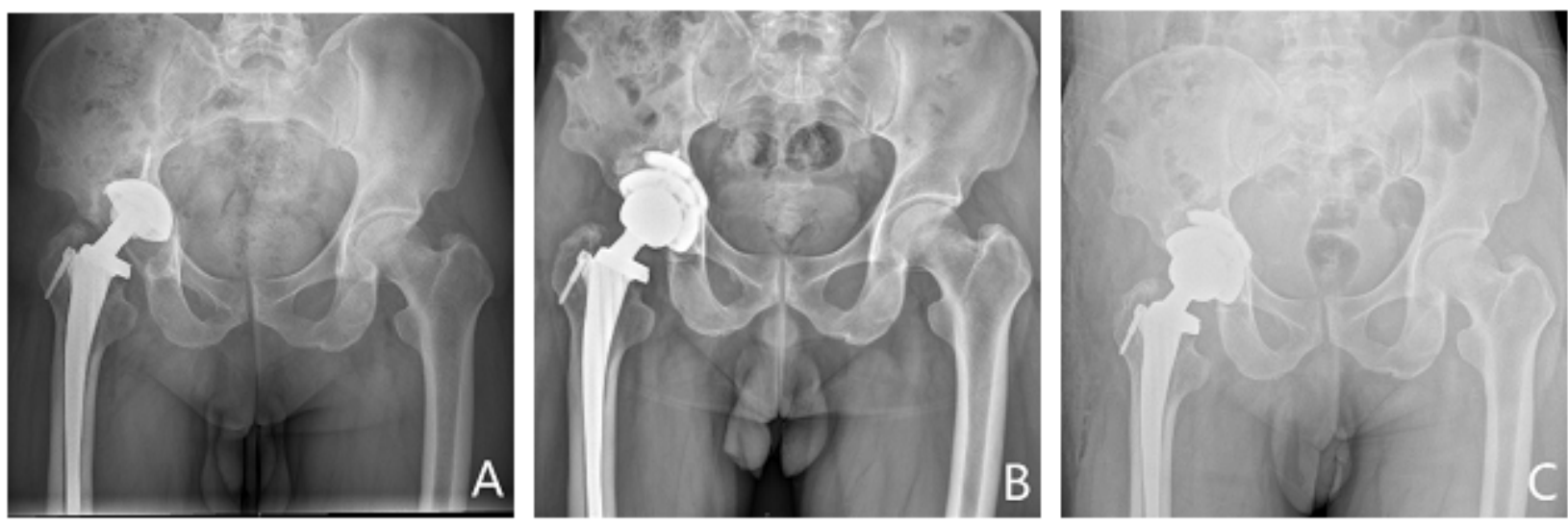

\section{Figure 3}

Five years after primary total hip arthroplasty, a 47-year-old man underwent revision surgery using a threedimensional printed trabecular titanium acetabular cup and augment due to aseptic loosening. (A) Preoperative radiograph showing a large acetabular defec. (B) Radiograph obtained at 1 day postoperatively. (C) Radiograph obtained at 25 months postoperatively showing the absence of a radiolucent line. 
A

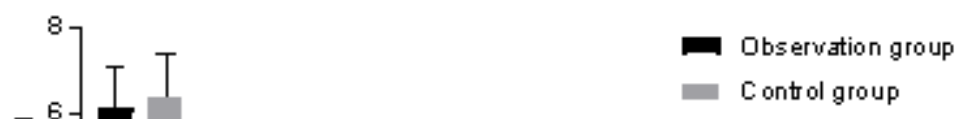

B

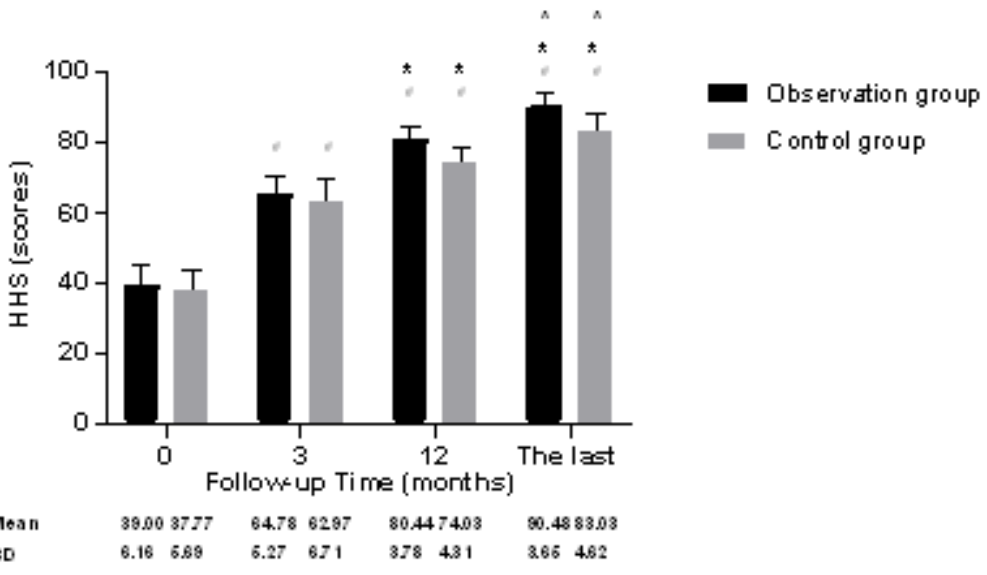

C

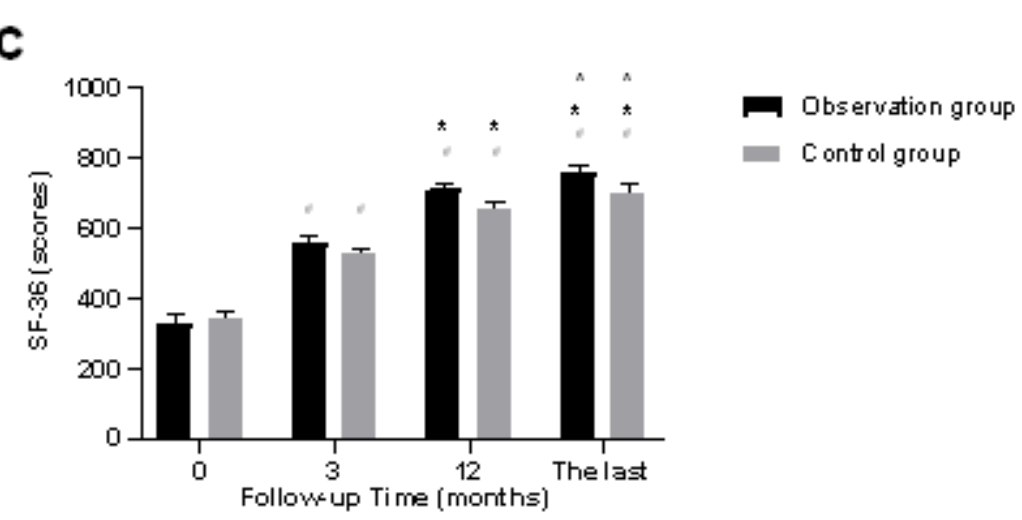

$\begin{array}{lllllllll}\text { Mean } & 6.11 & 6.32 & 3.96 & 3.99 & 2.26 & 2.18 & 114 & 1.21\end{array}$ $\begin{array}{lllllllll}3 D & 0.93 & 1.04 & 0.71 & 0.979 & 0.281 & 0.18 & 114 & 1.21 \\ & & & & 0.23 & 0.23 & 0.28\end{array}$

$\begin{array}{lllllllll}3 D & 6.16 & 6.89 & 6.27 & 671 & 378 & 431 & 3.66 & 4.82\end{array}$

Mean $\quad 326.44337 .03 \quad 66296624.12 \quad 711.61661 .29 \quad 76404700.71$

$\begin{array}{lllllllll}9 D & 31.09 & 29.78 & 22.28 & 21.0 & 16.21 & 26.19 & 2274 & 27.71\end{array}$

\section{Figure 4}

The graphs showed the changes of the VAS (A), HHS (B) and SF-36 (C) scores preoperatively, 3 and 12 months postoperatively, and at the last follow-up for patients in both groups. \# $p<0.05$ vs before surgery; ${ }^{*} p<0.05$ vs 3 months after surgery; ${ }^{\wedge} p<0.05$ vs 12 months after surgery. VAS: Visual Analogue Scale, HHS: Harris Hip Score, SF-36: Short Form 36 


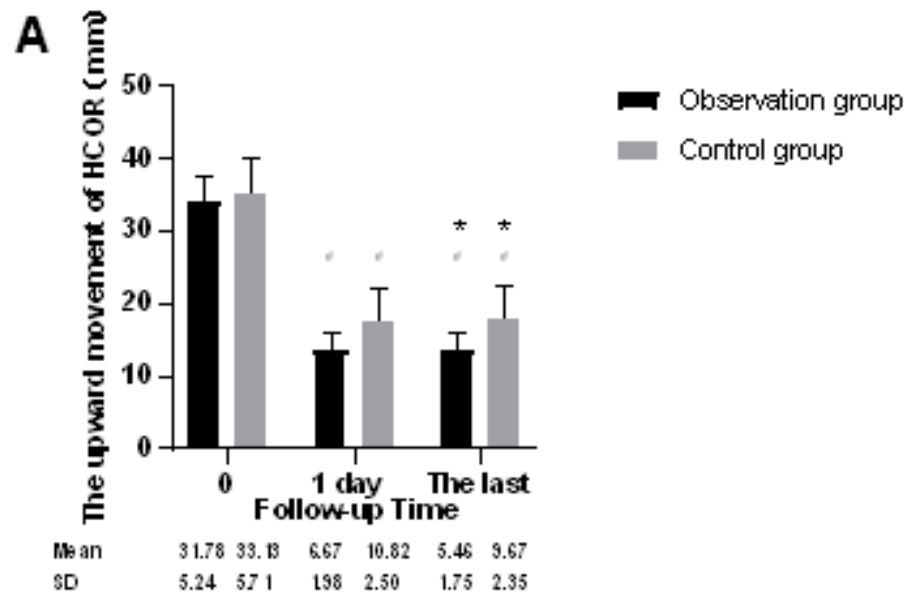

B

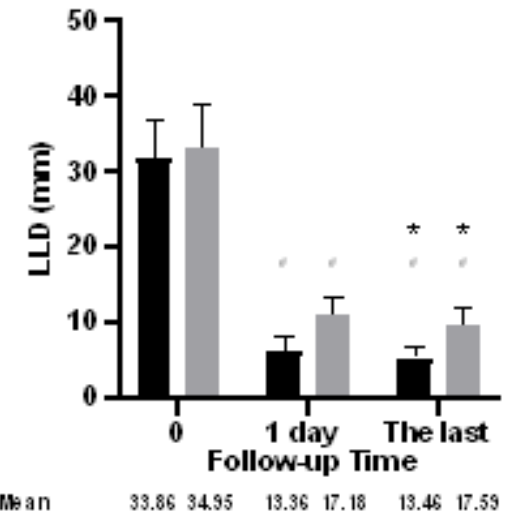

- Observation group

- Control growp

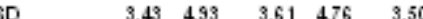

Figure 5

The graphs showed the changes of upward movement distance of the HCOR (A) and LLD (B) preoperatively, 1 day postoperatively, and at the last follow-up for patients in both groups. \# $p<0.05$ vs before surgery; * $p<0.05$ vs 1 day after surgery. HCOR: hip center of rotation, LLD: limb-length discrepancy 